\title{
A Study on Linked Lists
}

\author{
By \\ Nikhil Ranjan Nayak
}

April 10 $0^{\text {th }}, 2018$ 


\section{CERTIFICATE}

This is to certify that the project report entitled "A Study on Linked Lists" is submitted by Nikhil Ranjan Nayak of CSE-F for evaluation to Institute Of Technical Education And Research, Siksha 'O' Anusandhan University, Bhubaneswar, for the partial fulfillment for the degree of Bachelor of Technology in CSE is a record of original bonafide work carried out by me under the supervision and guidance of Mrs. Bishnupriya Panda. 


\section{INTRODUCTION}

\section{Linked List:}

A link list is one of the fundamental data structures and can be used to implement other data structures. It consists of a sequence of nodes, each containing (one or more as per requirement) data fields and one (or two) references (two for doubly link list) pointing to the next (or previous) nodes. We can use Link lists when we don't know the actual number of data. Link lists permit insertion and removal of nodes at any point in the list.

Field in node for Singly Link list:

$\rightarrow$ Data field: Data field of link list node contains useful information

$\rightarrow$ Reference (Link): Reference or Link field of one node contains reference of the other node.

- Linked list of items is arranged in order

- Size of linked list changes as items are inserted or removed

- Dynamic memory allocation is often used in linked list implementation

In this project I have implemented a singly linked list to create a simple smart ecard system (from the idea of Aadhaar Card). Original idea was to implement an RSA crypto algorithm to encrypt the database, but due to time issues I could not implement. 


\section{SINGLY LINKED LIST}

A singly linked list node is divided into two fields. The first field holds information about the node, and the second field holds the reference to the next node. When we link all nodes in some order then it becomes a singly linked list. In singly linked list we have only one reference to the next node, so in singly link list we can traverse in only one direction. The reference (link) points to the next node in the list, or to a null value (if it's a last node). We need one Head reference that points to the First node of the list.

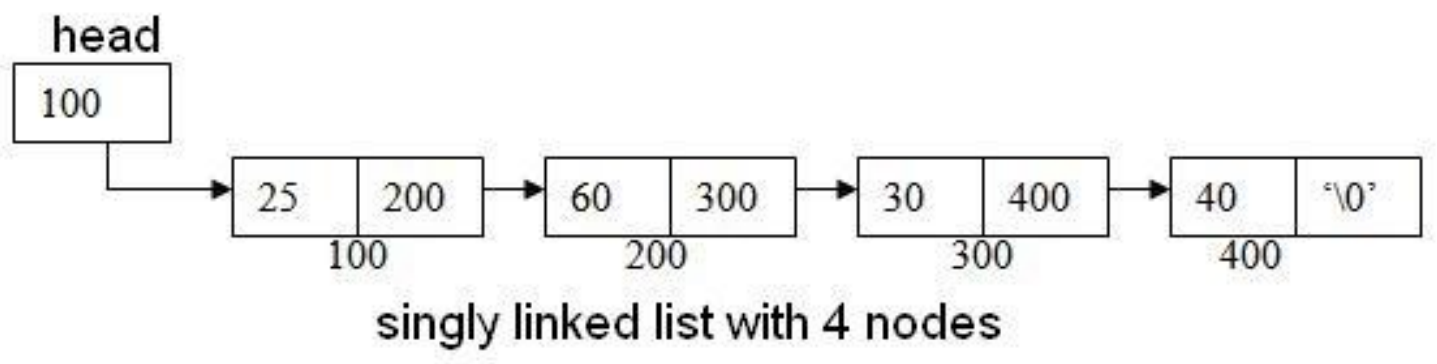




\section{Memory Representation of Singly Linked List}

Memory Representation of Singly Linked List is defined as:

typedef struct nodetype

\{

struct nodetype *prev;

int info;

struct nodetype *next;

\} node;

node *head, *tail;

\section{Structure of Singly Linked List}

A structure of a singly linked list is defined as:

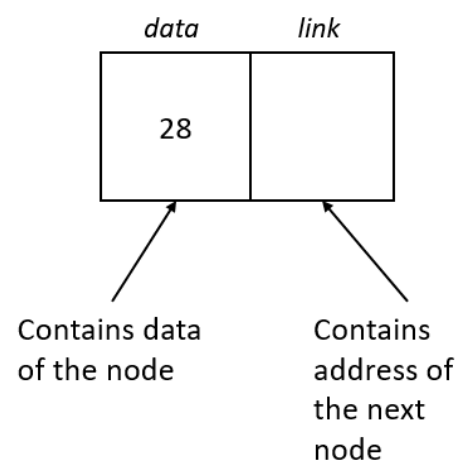

A node contains two parts:

1. Data: This part contains the data.

2. Link: This part contains the address of the next node. 


\section{Operations on Singly Linked List}

The following operations can be performed on the singly Linked List:

- Creating an Empty Linked List

- Traversals of List

- In-Order Traversal

- Reverse Order Traversal

- Inserting a node (or elements)

$\circ$ In the Beginning

- In the Middle (i.e. after first element)

- At the end of the list

- Deleting a node (or element) from list

$\circ$ From the Beginning

- From Middle (i.e. after first node)

- From the End

- Searching a node in the List

- Finding the largest element (or Node)

- Finding the smallest element (or Node)

- Counting the total Nodes in the List

- Deleting Entire Linked List 


\section{TRADE OFFS}

As with most choices in computer programming and design, no method is well suited to all circumstances. A linked list data structure might work well in one case, but cause problems in another. This is a list of some of the common trade-offs involving linked list structures.

\begin{tabular}{|l|l|l|l|l|l|l|}
\hline & \multicolumn{1}{|c|}{ Linked list } & Array & $\begin{array}{c}\text { Dynamic } \\
\text { array }\end{array}$ & $\begin{array}{c}\text { Balanced } \\
\text { tree }\end{array}$ & \multicolumn{1}{c|}{$\begin{array}{c}\text { Random } \\
\text { access list }\end{array}$} & $\begin{array}{c}\text { Hashed array } \\
\text { tree }\end{array}$ \\
\hline Indexing & $\Theta(n)$ & $\Theta(1)$ & $\Theta(1)$ & $\Theta(\log n)$ & $\Theta(\log n)^{[5]}$ & $\Theta(1)$ \\
\hline $\begin{array}{l}\text { Insert/delete at } \\
\text { beginning }\end{array}$ & $\Theta(1)$ & $\mathrm{N} / \mathrm{A}$ & $\Theta(n)$ & $\Theta(\log n)$ & $\Theta(1)$ & $\Theta(n)$ \\
\hline Insert/delete at end & $\begin{array}{l}\Theta(1) \text { when last } \\
\text { element is known; } \\
\Theta(n) \text { when last } \\
\text { element is unknown }\end{array}$ & $\mathrm{N} / \mathrm{A}$ & $\begin{array}{l}\Theta(1) \\
\text { amortized }\end{array}$ & $\Theta(\log n)$ & $\Theta(\log n)$ updating & $\Theta(1)$ amortized \\
\hline $\begin{array}{l}\text { Insert/delete in middle } \\
\text { Wasted space }\end{array}$ & $\begin{array}{l}\Theta(n) \\
\text { (average })\end{array}$ & $\mathrm{N} / \mathrm{A}$ & $\Theta(n)$ & $\Theta(\log n)$ & $\Theta(\log n)$ updating & $\Theta(n)$ \\
\hline
\end{tabular}




\section{REFERENCES}

- Tutorials Point

○ https://www.tutorialspoint.com/data_structures_algorithms/

- HackerEarth

- https://www.hackerearth.com/practice/data-structures/linked-list/singlylinked-list/tutorial/

- Wikipedia

○ https://en.wikipedia.org/wiki/Linked_list 